\title{
Research Paper: \\ Prevalence of Premenstrual Syndrome, Premenstrual Dysphoric Disorder and Associated Factors Among Female High School Students
}

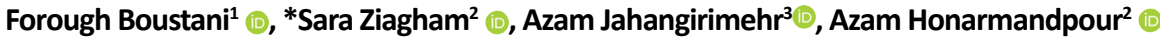

1. Student Research Committee, Shoushtar Faculty of Medical Sciences, Shoushtar, Iran.

2. Department of Midwifery, Shoushtar Faculty of Medical Sciences, Shoushtar, Iran.

3. Department of Health, Shoushtar Faculty of Medical Sciences, Shoushtar, Iran.

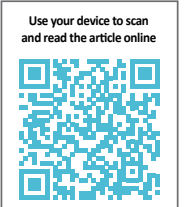

Citation Boustani F, Ziagham S, Jahangirimehr A, Honarmandpour A. Prevalence of Premenstrual Syndrome, Premenstrual Dysphoric Disorder and Associated Factors Among Female High School Students. The Journal of Qazvin University of Medical Sciences. 2019; 23(4):332-341. https://doi.org/10.32598/JQUMS.23.4.332

doi https://doi.org/10.32598/JQUMS.23.4.332

Received: 24 Feb 2019 Accepted: 25 Jun 2019 Available Online: 01 Oct 2019

Keywords: Premenstrual syndrome, Premenstrual dysphoric disorder, Prevalence, High school students

\section{A B STR ACT}

Background Premenstrual syndrome (PMS) is a common psychosomatic disorder linked to menstruation in women at gestational age and can lead to absence from work and academic failure.

Objective This study aimed to determine the prevalence and severity of PMS, premenstrual dysphoric disorder (PMDD) and associated factors among the female high school students.

Methods This descriptive-analytical study was carried using randomized classification method on 368 female high school students in Shoushtar, Iran in 2016. Data were collected by a demographic form and Premenstrual Symptoms Screening Tool. Collected data were analyzed using Kolmogorov-Smirnov test, Chi-squared test, binomial test, Mann-Whitney test and Spearman correlation test at a significance level of $\mathrm{P}<0.05$.

Findings The Mean \pm SD age of participants was $16.3 \pm 0.87$ years. The prevalence of PMS in students was $53.5 \%$ and for PMDD was $12.22 \%$. The most prevalent symptoms were mood, behavioral and physical symptoms. The frequency of PMS was higher in students with a positive family history.

Conclusion The prevalence of PMS in high school female students was relatively high. Since this problem can interfere with one's personal, social and academic activities, an educational program at schools and informing students in this regard can be helpful and effective in promoting the health and young girls.

\section{Extended Abstract}

\section{Introduction}

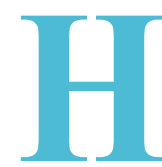

ealth has been a top priority for women's rights in recent years, because in addition to their emotional role in the family, their occupational and social roles in society have become more prominent [1]. One of the psychosomatic issues associated with the reproductive system function in women is premenstrual syndrome (PMS). PMS is defined as recurrent mood, emotional, and physical symptoms that occur during luteal menstrual phase which is solved with the onset of menstruation immediately or a few days after it [2]. According to different studies conducted around the world, the prevalence of PMS has been reported to be $40 \%$ in Europe, $85 \%$ in Africa, $46 \%$ in Asia, and $60 \%$ in South America [7].

\section{* Corresponding Author: \\ Sara Ziagham}

Address: Department of Midwifery, Shoushtar Faculty of Medical Sciences, Shoushtar, Iran.

Tel: +98 (916) 6189725

E-Mail: saraziagham@yahoo.com 
Its prevalence among adolescents and youngsters in Iran has been increased from $16 \%-78.4 \%[3,8]$. The prevalence of this syndrome in a society varies depending on the factors such as culture, attitude, age, exercise, diet, and underlying diseases. It has high effects on individual and social functioning of patients with PMS. Considering the important individual and social role of women in society, and given that no study has been conducted to investigate the prevalence of PMS in Shoushtar, Iran, the present study was aimed to investigate the prevalence of PMS and premenstrual dysphoric disorder (PMDD) and the factors associated with them.

\section{Materials and Methods}

This descriptive-analytical study was carried out on 368 female students studying at public high schools of Shoushtar, Iran in 2016. The samples were selected from 15 high schools by stratified random sampling technique. Data were collected by a demographic form (surveying age, body mass index (BMI), mother's age and job, age at menarche, and length of menstrual period), and the premenstrual symptoms screening tool (PSST) in Persian to determine the PMS and PMDD prevalence. The PSST consisted of 19 items in two sections rated as $0=$ never, $1=$ mild, $2=$ moderate, and $3=$ severe. The collected data were analyzed in SPSS V. 21 software using descriptive statistics and Kolmogorov-Smirnov test of normality, chisquare test of agreement between qualitative variables, and Man-Whitney test for comparison of quantitative variables between two study groups. The difference was considered significant if $\mathrm{P}<0.05$.

\section{Results}

Students had a Mean \pm SD age of $16.3 \pm 0.87$ years. Their age at menarche ranged 9-16 years with a Mean \pm SD value of $12.68 \pm 1.19$ years. They reported the length of menstrual period as 2-10 days with a mean value of $5.9 \pm 1.45$ days; for $319(87.4 \%)$ of them, mother's job was housekeeping and for others, the status was "employed"; 226 (61.41\%) had physical activity and 142 (38.59\%) had no activity; and 143 (39.4\%) were using chemical medicines to relieve their pain. In this study, family history of PMS $(\mathrm{P}=0.017)$ and mother's job $(\mathrm{P}=0.046)$ had a significant effect on the occurrence of PMS.

No significant difference was found between the two groups in terms of age, height, BMI, age at menarche, and length of menstrual period $(\mathrm{P}>0.05)$. From among participants, 197 (53.5\%) had the signs and symptoms of PMS, of whom $20(10.2 \%)$ had mild symptoms, 89 (45.2\%) had moderate symptoms, and $88(44.7 \%)$ had severe symp- toms. Moreover, 45 (12.22\%) suffered from PMDD. The most common symptoms included nervousness/irritability $(\mathrm{n}=151,82.6 \%)$, muscle/joint pain $(\mathrm{n}=109,59.9 \%)$, and relationship with family $(\mathrm{n}=138,75.8 \%)$.

\section{Conclusion}

Our results reported the PMS prevalence of $49.72 \%$ and PMDD prevalence of $12.22 \%$. Studies have reported different rates of PMS prevalence in different countries. For example, it is 38\% among high school students in Iran (Kerman) and 11\% in Switzerland, 92\% among Chinese women, $64.6 \%$ among Japanese female adolescents, 53\% for the Pakistani population, and $30.4 \%$ in the French population $[8,9,14]$. The prevalence of this syndrome is $67.7 \%$ for the Iranian women working in factories and $98.2 \%$ for the Iranian female college students [15]. Ahmadi et al. in a study on the female high school students of Ilam city, in Iran reported a prevalence rate of $36.84 \%$ for PMS and $11.65 \%$ for PMDD [23]. Our results are consistent with the results of above mentioned studies.

Family history of PMS was observed in $28.3 \%$ of participants in our study. There is a considerable relationship between the mothers and daughters and between sisters in terms of menstrual symptoms, indicating that the mother's attitude is most likely to influence her daughter's response to the disease. Positive family history increases the possible occurrence of PMS [5]. The most prevalent physical signs of PMS in the present study were muscle pain, headache, and flatulence and the least common signs were breast pain and overweight. In the previous studies $[4,17,18]$, the most common physical signs were reported muscle pain and flatulence, which are in agreement with the results of our study. The most prevalent mood symptoms in the current study were anger, fatigue/shortage of energy, and difficulty concentrating and the least common symptoms were loss of interest in working and insomnia.

\section{Ethical Considerations}

\section{Compliance with ethical guidelines}

This study was approved by the Research Ethics Committee of Shoushtar Faculty of Medical Sciences (Code: IR.AJUMS.REC.1395. 731).

\section{Funding}

This study was extracted from a research project approved by the Shoushtar Faculty of Medical Sciences, Shoushtar. 


\section{Authors' contributions}

Conceptualization: Forough Boustani and Sara Ziagham; Writing and data analysis: Forough Boustani, Azam Jahangirimehr and Azam Honarmandpour; Editing, supervision and project administration: Sara Ziagham; Initial draft preparation by Forough Boustani.

Conflicts of interest

The authors declare no conflicts of interest.

Acknowledgements

The authors would like to thank the Deputy for Research and Technology of Shoushtar University of Medical Sciences, and the students participated in the study for their valuable cooperation and support. 


\title{
شيوع علائم بالينى سندرم يبش از قاعدتى و اختلال ملال يبش از قاعدتى و عوامل مرتبط با آن، در دانش آموزان دبيرستانى شهر شوشتر سينر
}

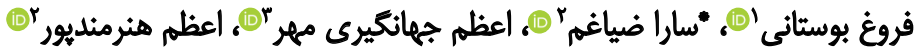 \\ 1. كميته تحقيقات دانشجويى، كروه مامايي، دانشكده علوم بزُشكى شوشتر، شوشتر، ايران.

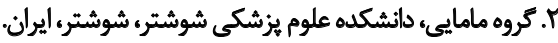

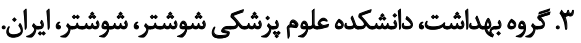

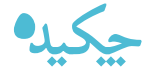

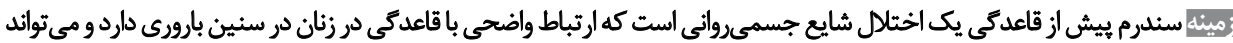
به غييت از محل كار و يا افت تحصيلى منجر شوده.

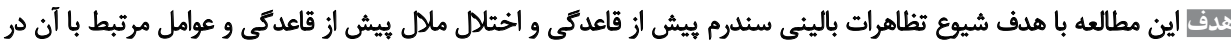

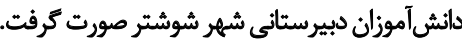

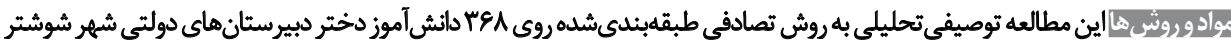

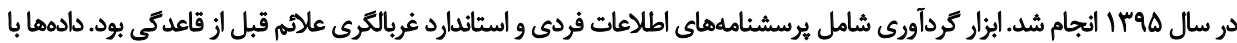

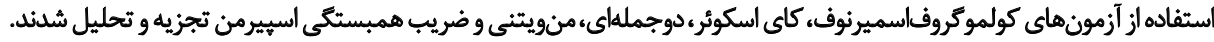

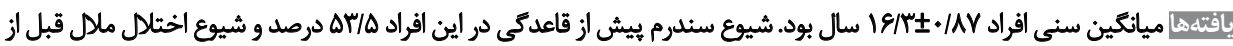

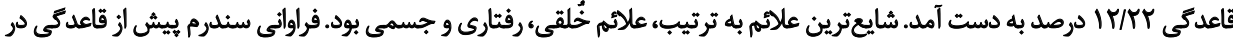

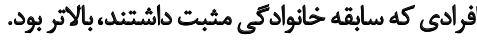

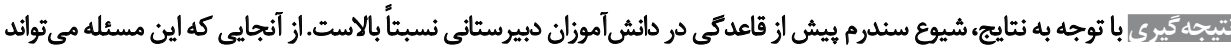

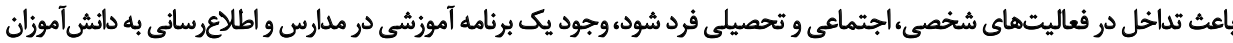

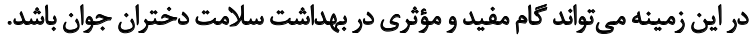

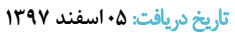

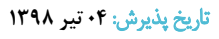

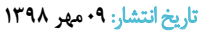

مي تواند در روابط و عرصههاى مختلف اجتماعى و شغلى اثركذار

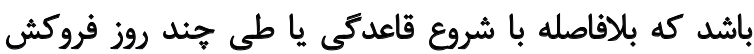

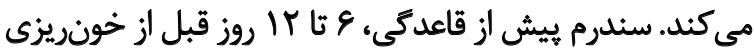

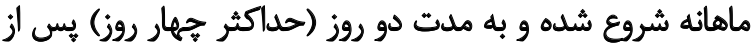

شروع خونريزى تداوم دارد [ب].

نظريههاى عصبى هورمونى هيجكدام بهتنهايى نمى توانئد تمام

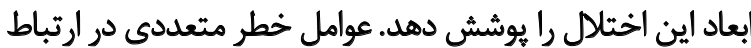

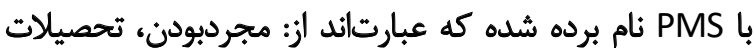

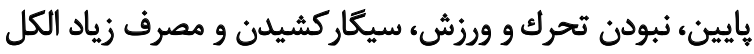

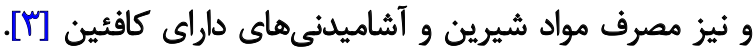



2. Premestrual Disphoric Disorder (PMDD) dato

سلامتى در سالهاى اخير، يكى از الولويتهاى حقوق زنان

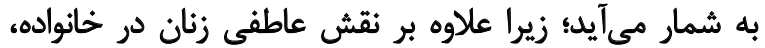

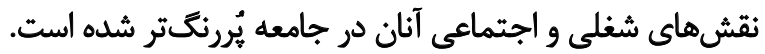

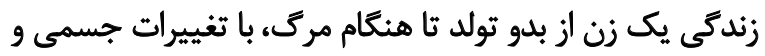

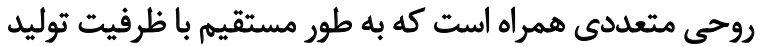

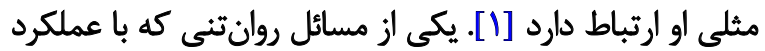

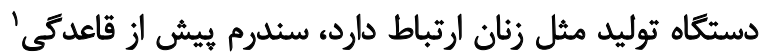

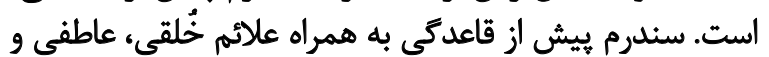

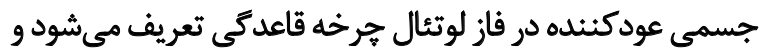

1. Premenstural Syndrom (PMS)

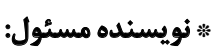

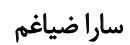

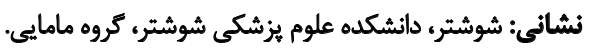

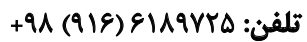

$$
\begin{aligned}
& \text { رايانامه: saraziagham@yahoo.com }
\end{aligned}
$$


جسمى و انزواى اجتماعى را در بـى دارد. همجنين باعث افزايش

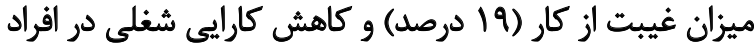

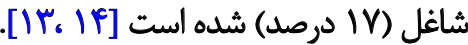
شيوع زياد اين سندرم در جامعه با توجه به عواملى نظير

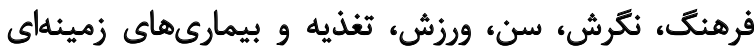

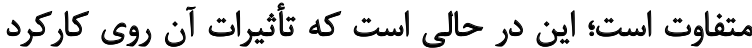

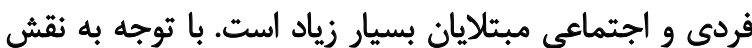

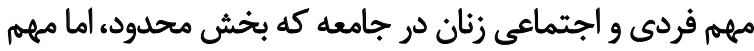

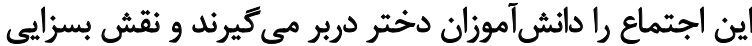

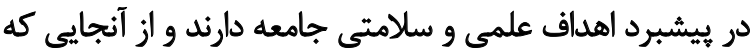

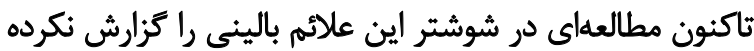

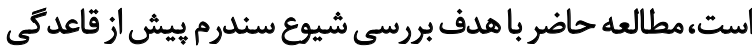
و اختلال ملال بيش از قاعدكى و عوامل مرتبط با آن طراحى شد. شئد.

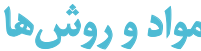

اين مطالعه توصيفىتحليلى روى ^وع دانش آموز دختر در حال

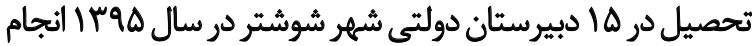

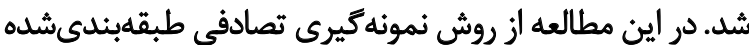

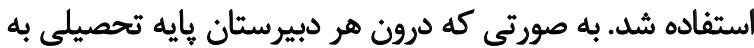

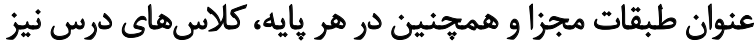

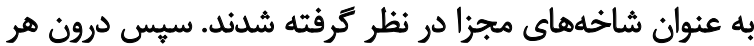

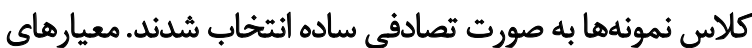

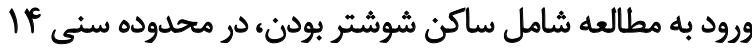

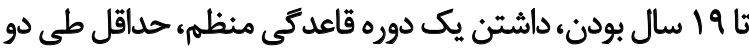

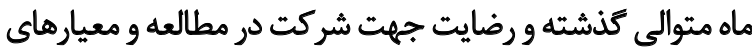

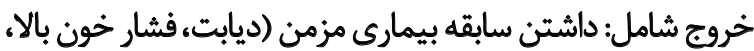

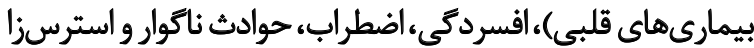

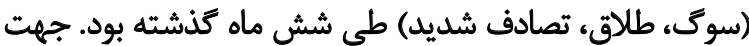

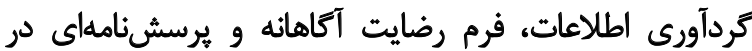

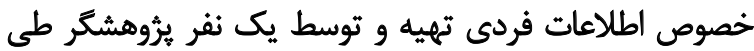

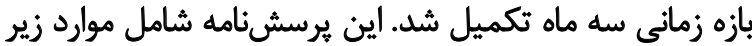

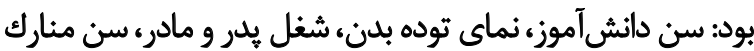

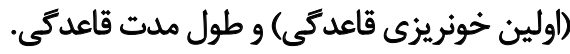

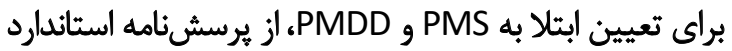

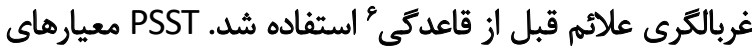

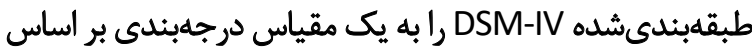

شدت بيمارى تبديل مي كند [10]

در مطالعه سيدبازى و همكاران ياياييى يرسشنامه با با استفاده



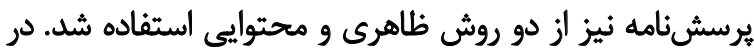

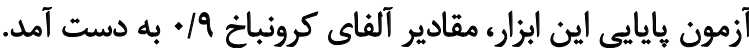

6. Premenstrual symptoms screening tool (PSST)
سندرم بيش از قاعدكى در نظر گرفته شده كه باعث اختلال

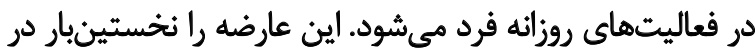

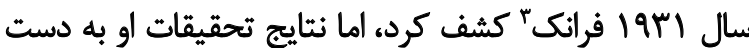

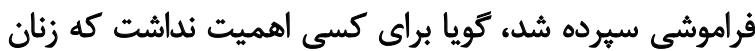

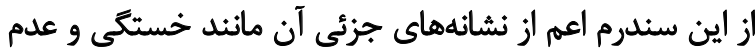

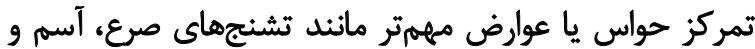

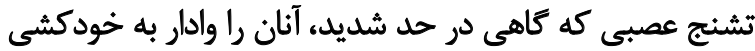

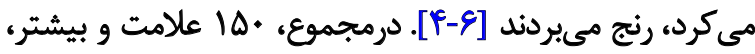

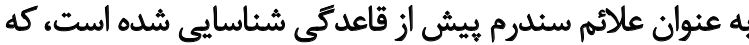

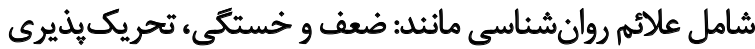

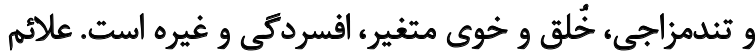

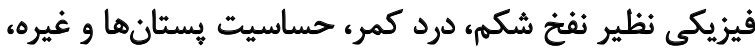

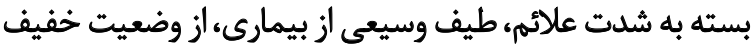

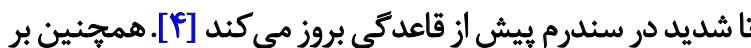

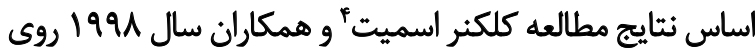

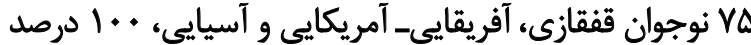

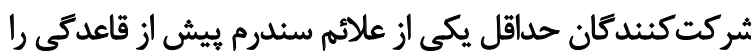

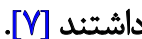

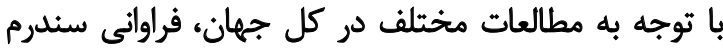

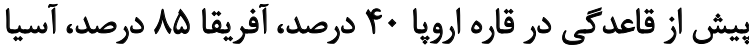
هو

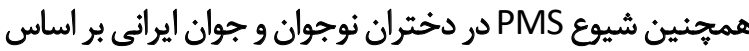

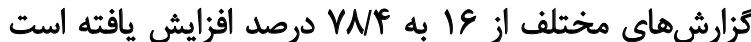

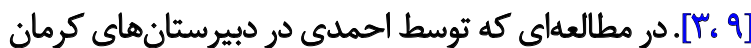

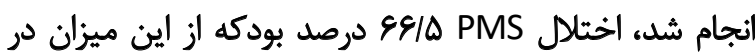

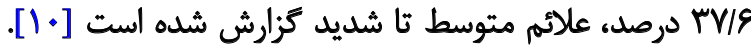

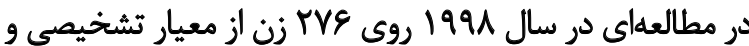

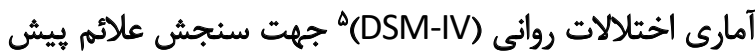

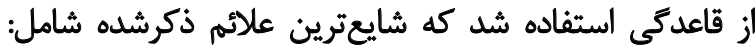

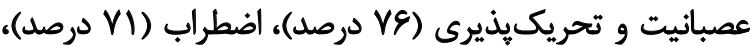

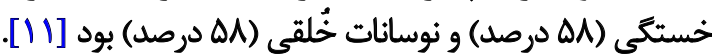

طى مطالعهاى كه توسط اميرى فراهانى و همكاران در سال

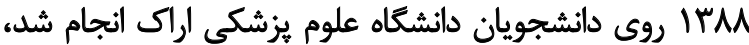



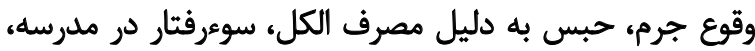

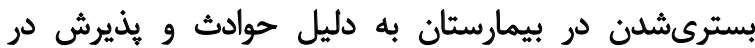

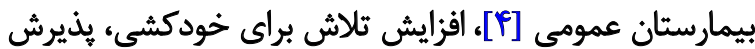

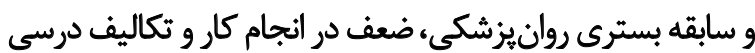

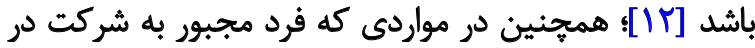

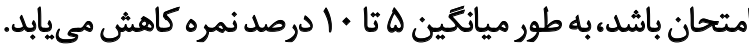

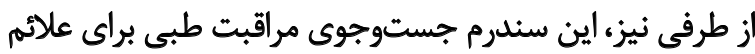

5. Diagnostic and Statistical Manual of Mental Disorders, $4^{\text {th }}$ Edition 


$$
\text { بود (P=. ) }
$$

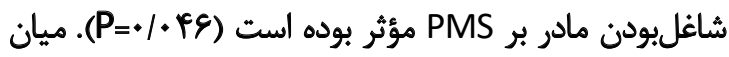

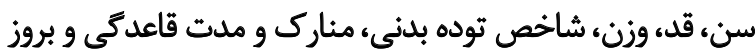

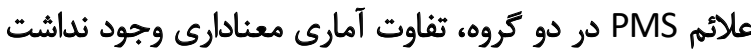

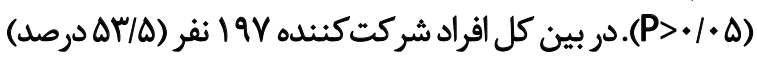

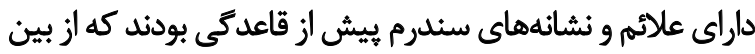

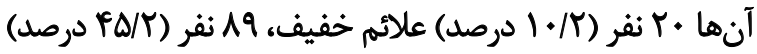

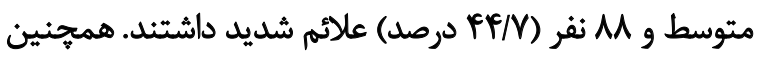

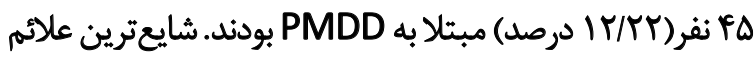

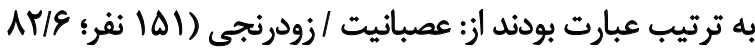

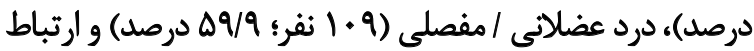

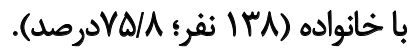

\section{بحث و تئيجنا كَيرى}

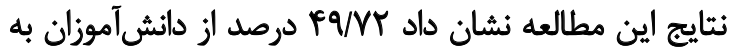

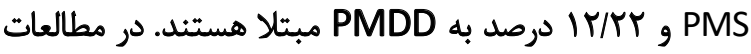

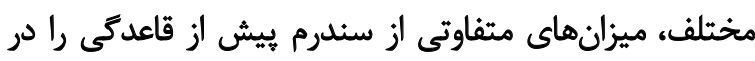

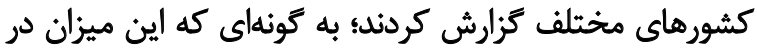

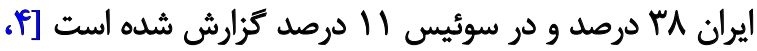

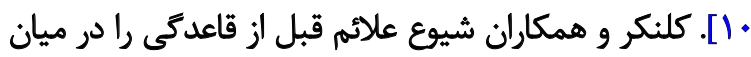

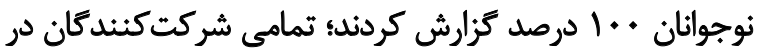

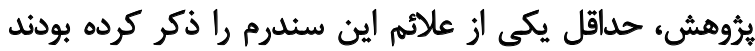

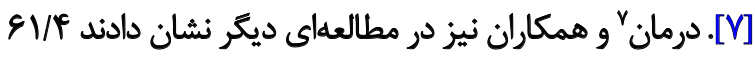

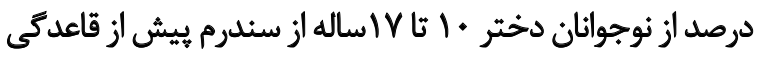

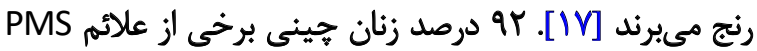

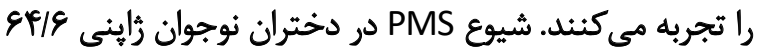

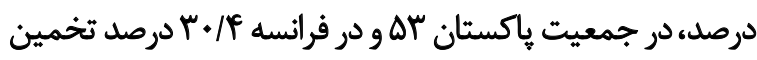

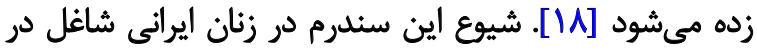

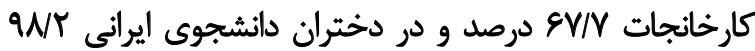

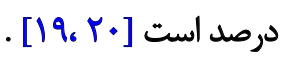

به طور كلى مىتوان اين تفاوتها را با تنوع در روش مطالعه،

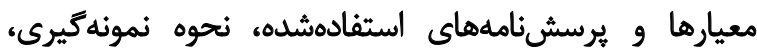

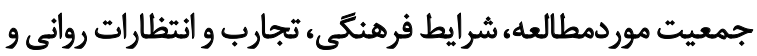

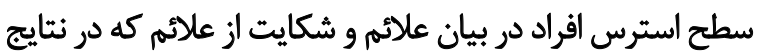

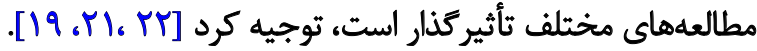

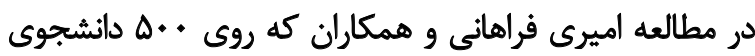

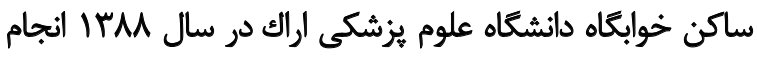

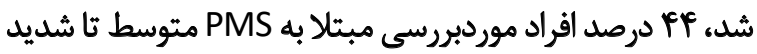

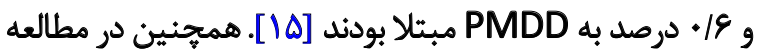

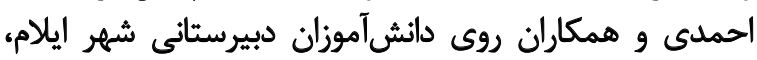

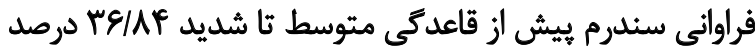

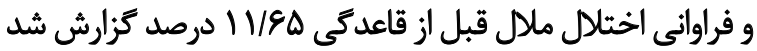

\section{Derman}

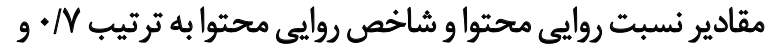

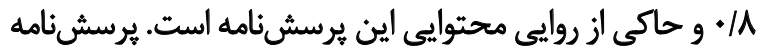

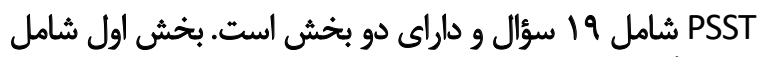

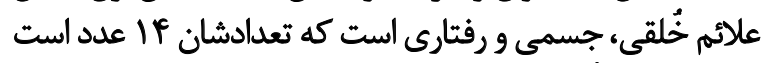

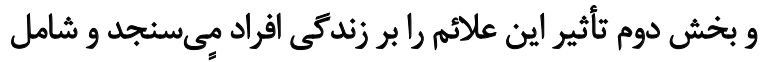

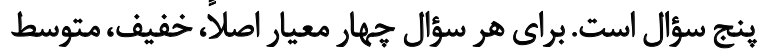

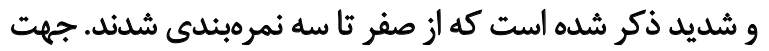

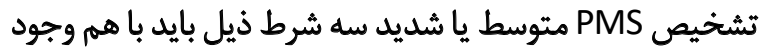

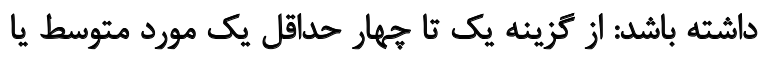

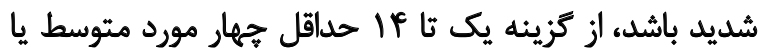

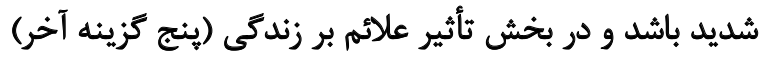

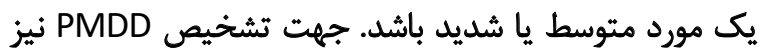

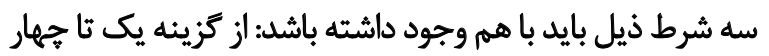

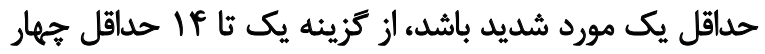

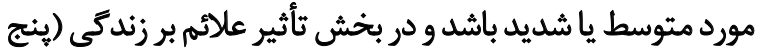
كزينه آخر) يك مورد شديد باشد ياشد [19].

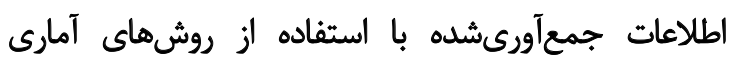

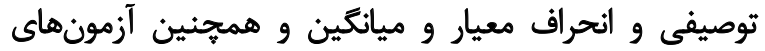

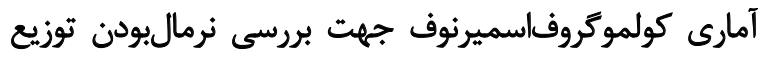

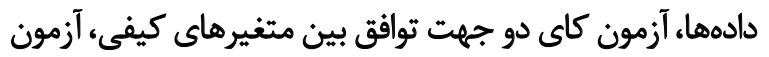

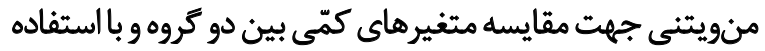

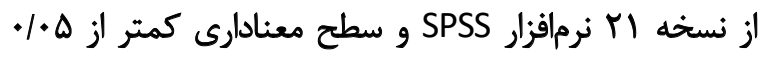

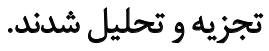

Lاب

If اين مطالعه روى

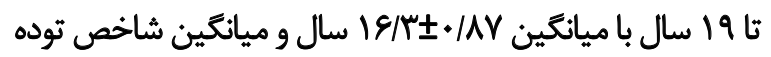

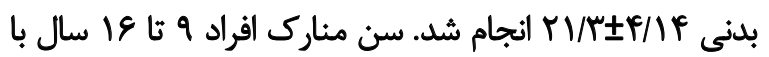

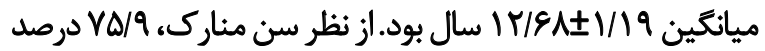

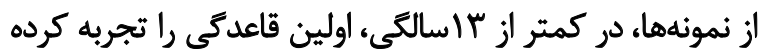



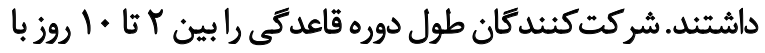

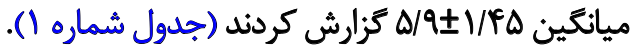

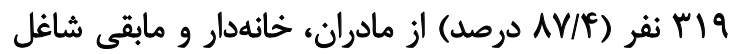

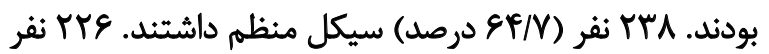
(F//FI)

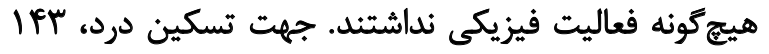

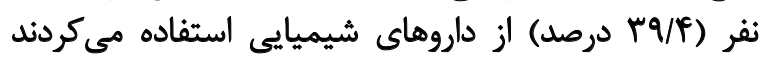

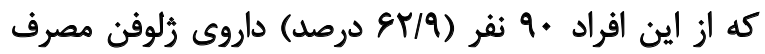

$$
\text { مى كردند (جدول شماره (1). }
$$

توزيع متغيرهاى سن، شاخص توده بلدنى، سن منارك و ومدات

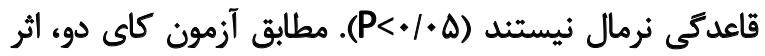

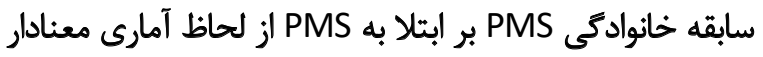


جدول ا. مقايسه توزيع فراوانى برخى از مشخصات فردى دختران در بين دو كروه وجود يا نبود سندرم ييش از قاعدكى

\begin{tabular}{|c|c|c|c|c|}
\hline \multirow{2}{*}{ (أزمون معنى داري) } & \multicolumn{2}{|c|}{ تعداد (درصد) } & \multirow{2}{*}{ كروه } & \multirow{2}{*}{ مشحصات فردى } \\
\hline & نبود PMS & وجود PMS & & \\
\hline \multirow{5}{*}{ - /ADQ } & $9(4+/ 9)$ & $\pi(\Delta \nabla /)$ & بيىسواد & \multirow{5}{*}{ تحصيلات مادر } \\
\hline & $P Q(P V / 9)$ & $P q(\Delta T / N)$ & ابتدايع & \\
\hline & D) $(F E / f)$ & $\Delta 9(\Delta r / 9)$ & زيردييلم & \\
\hline & $r q(8+/ \pi)$ & a) $(\Delta \& M)$ & دييلم & \\
\hline & $r \Delta(\Delta Y / M)$ & $M(r v / 9)$ & دانشكاهى & \\
\hline \multirow[b]{2}{*}{$.1+48$} & $M(\Delta N Y)$ & $19(81 / 4)$ & شاغل & \multirow[b]{2}{*}{ شغل مادر } \\
\hline & $|T|(N T / T)$ & $\operatorname{IYA}(\Delta \Delta / A)$ & خانهدار & \\
\hline \multirow{3}{*}{$.1 \% 1 V^{*}$} & $\Delta V(T q / T)$ & $M(8+M)$ & دارد & \multirow{3}{*}{ سابقه خانوادكى } \\
\hline & & & & \\
\hline & $\| F(\Delta V / I)$ & $1.9(4 N 9)$ & ن ندارد & \\
\hline \multirow[b]{2}{*}{. ITEY } & 118 (NNV) & $\mathbb{M r}\left(\Delta / \mathbb{N}^{*}\right)$ & دارد & \multirow[b]{2}{*}{ سيكل منظهم } \\
\hline & $\Delta \Delta(N T / M)$ & $V \Delta(\Delta V M)$ & ندارد & \\
\hline \multirow{5}{*}{.1 .98} & $10(F \Delta / \Delta)$ & $M(r \Delta / \Delta)$ & كياهى & \multirow{5}{*}{ داروى مصرفى } \\
\hline & $\Delta A(\%+18)$ & $N \Delta(\Delta \vee / 4)$ & شيميايى & \\
\hline & & & & \\
\hline & If $(F V / A)$ & $M(R / M)$ & هردو & \\
\hline & $\operatorname{Ar}(\Delta F / M)$ & $8 \Lambda(\% \Delta / \%)$ & هيجكدام & \\
\hline \multirow{3}{*}{.$/ p n^{e}$} & $1 . \Delta(E \& / \Delta)$ & $|T|(\Delta r / \Delta)$ & دارد & \multirow{3}{*}{ فعاليت فيزيكىى } \\
\hline & & & & \\
\hline & ef (FV/F) & $n(\Delta r / \varepsilon)$ & ندارد & \\
\hline
\end{tabular}

\begin{tabular}{|c|c|c|c|}
\hline سطح معنى دارى (أزمون منويتنى) & \multicolumn{2}{|c|}{ مياتكين ثانحراف معيار } & متغير \\
\hline ./apr & $\mid \varnothing / \pi \cdot \pm \cdot / A \Delta$ & $18 / 7 q \pm \cdot / 19$ & سن اقراد \\
\hline.$/ 994$ & $|\&| / \Delta \& \pm g / m q$ & $\mid 91 / 1 \mathrm{~A} \pm 9 \cdot 9$ & قد افراد \\
\hline.$/ 9.1$ & $\Delta \Delta / \Delta F \pm I I / N$ & $\Delta \Delta / f \& \pm 11 / / 2 q$ & 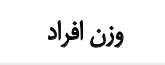 \\
\hline.$/ P \Delta V$ & 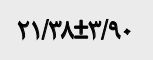 & 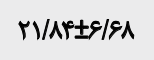 & شاخص توده بدنى \\
\hline.$/ 101$ & $\| T M \pm I / I V$ & $\mid r / F \Delta \pm 1 / T r$ & 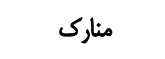 \\
\hline .1014 & $\Delta / Q \Psi \pm I / \& \varepsilon$ & $\Delta / \neg \Delta \pm 1 / F \Delta$ & مدت قاعدكى \\
\hline
\end{tabular}

مثبت، احتمال بروز سندرم بيش از قاعدكى را بيشتر مي كند

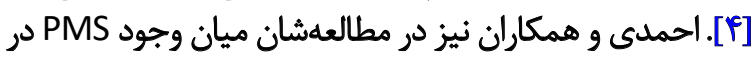

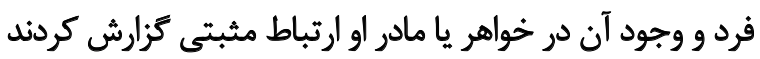

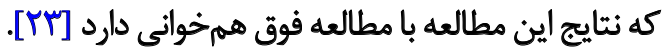
شايعترين علامت جسمى در مطالعه حاضر، درد عضلانى،

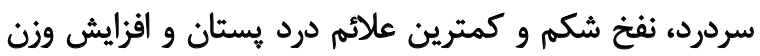

كه نتايج اين مطالعه با مطالعات فوق همخوانى دارد [TM].

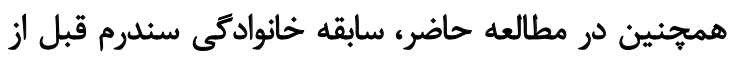

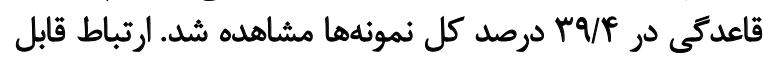

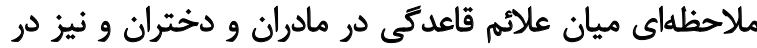

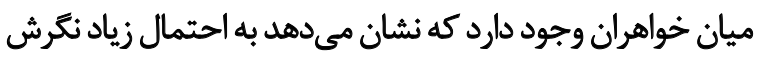

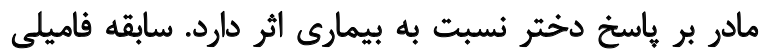




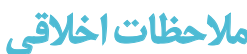

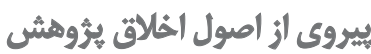

اين مقاله منتج از طرح تحقيقائى تصويبشده در معاونت

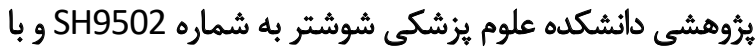

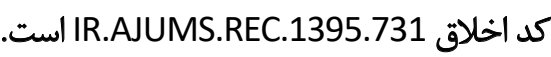

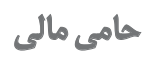

اين مقاله منتج از طرح تحقيقاتى تصويبشده در كميته

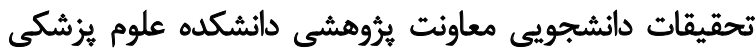

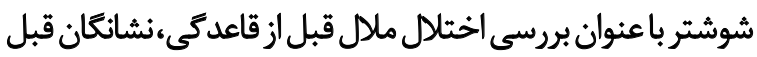

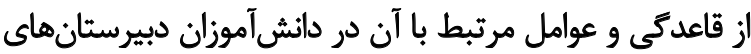

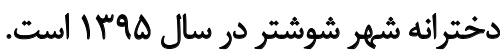

$$
\text { مشاركت ثويسند تان }
$$

انتخاب موضوع مطالعه و جمعبندى مطالب: ساراضياغم و فروغ بوستانى؛ نكارش و تحليل دادهها: فروغ بوستاني، اعظهم جهانيكيرى

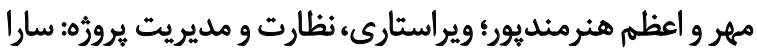
ضياغم: بيشنويس مقاله: فروغ بوستانى.

$$
\text { ت تعارض مثافع }
$$

بنابر اظهار نويسندكان اين مقاله تعارض منافع ندارد.

$$
\text { تشيكر و قبدرواني تي }
$$

بدينوسيله از معاونت محترم آموزش و تحقيقات و شوراى

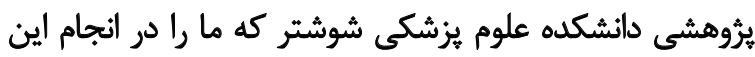
تحقيق يارى كردند تشكر و قدردانى مي كنيه.
بود. در مطالعهاي كه توسط نجفى شرجآباد و همكاران انجام

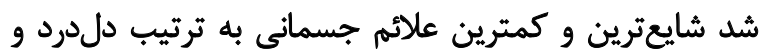

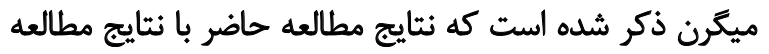

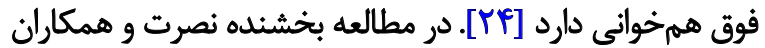

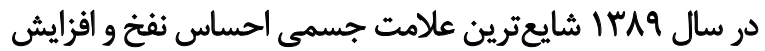

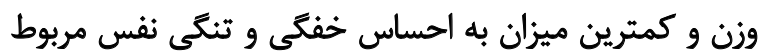

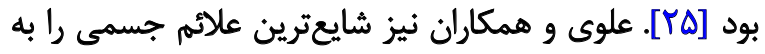

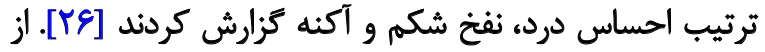

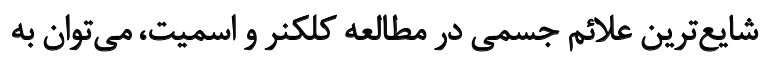

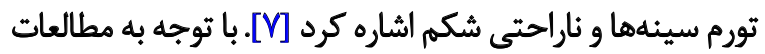

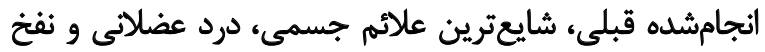

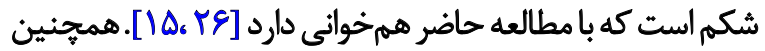

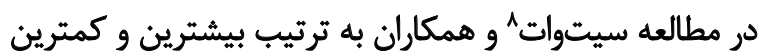

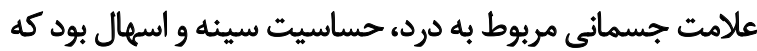

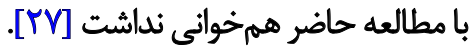

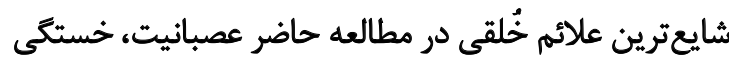

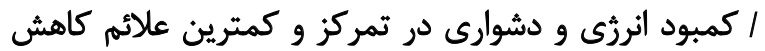

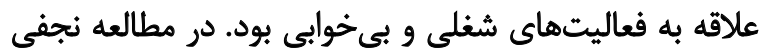

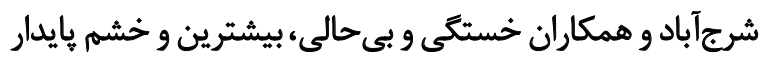

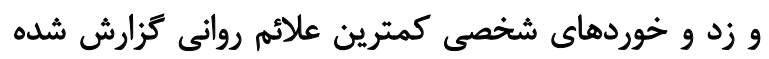



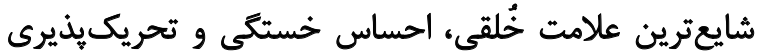

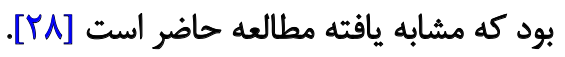

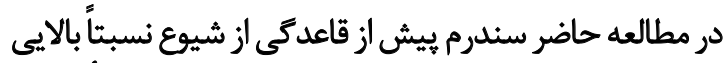

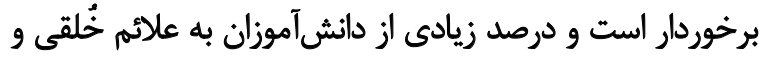

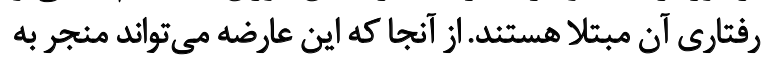

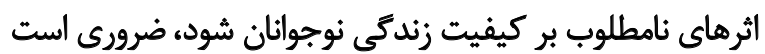

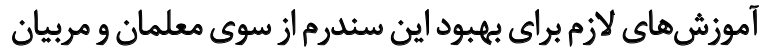

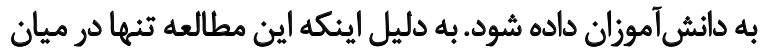

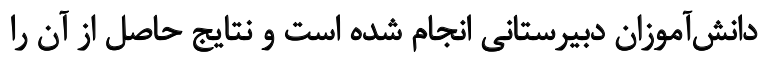

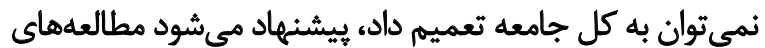

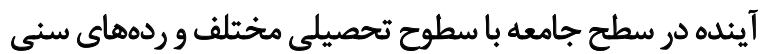

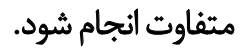
از نقاط قوت اين مطالعه مىثوان به اين نكته اشاره كرد كه

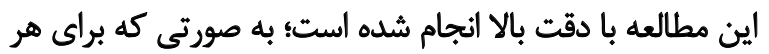

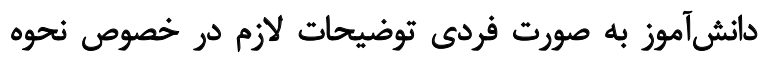

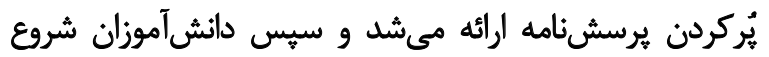

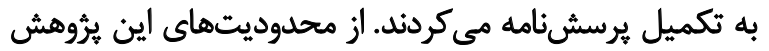

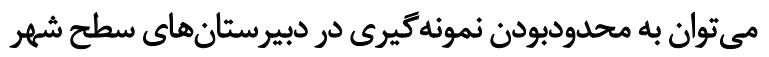

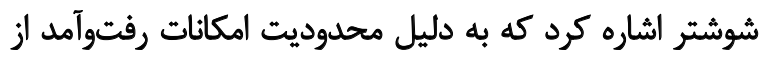
انتخاب مدارس حومه شهر شوشتر صرف نظر شدر شدرد 


\section{References}

[1] Jafarnejad F, Fadardi JS, Najafi MN, Shakeri Z. Evaluation the relationship between stress and the risk of premenstrual syndrome. Iran J Obs Gynecol Fertil. 2013; 16(76):8-11. [In Persian]

[2] Atashzade Shourideh F, Abbaszadeh A, Borhani F, Falahi Khoshknab $\mathrm{M}$, Firozkohi MR, Navabi Rigi SH, et al. The impact of valerian root extract on mood and behavioral symptoms severity in premenstrual syndrome. Med Surg Nurs J. 2014; 3(2):71-6. [In Persian]

[3] Tatari F, Torkamani F, Shakeri J, Rezaie M, Hosseini M, Amirian $M$, et al. Evaluation of the frequency of premenstrual dysphoric disorder and premenstrual syndrome in students of girls' high schools of Kermanshah-Iran. Eur Psy. 2011; 26:1688. [In Persian] [DOI:10.1016/S0924-9338(11)73392-2]

[4] Speroff L, Fritz MA. Clinical gynecologic endocrinology and infertility. New York: Lippincott Williams \& Wilkins; 2004.

[5] Varney H, Kriebs MJ, Geger IC. Varney's midwifery. London: Jones \& Bertlett Publ; 1997.

[6] Berkowitz RS, Barbieri RL, Dunaif AE, Kistner RW, Ryan KJ. Kistner's gynecology: Principle and practice. Philadelphia: Mosby; 1999.

[7] Cleckner-Smith CS, Doughty AS, Grossman JA. Premenstrual symptoms. Prevalence and severity in an adolescent sample. J Adolesc Health. 1998; 22(5):403-8. [DOI:10.1016/S1054-139X(97)00239-5]

[8] Direkvand Moghadam A, Kaikhavani S, Sayehmiri K. The worldwide prevalence of premenstrual syndrome: A systematic review and meta-analysis study. Iran J Obst Gynaecol Fertil. 2013; 16(65):8-17. [In Persian]

[9] Bakhshani NM, Mousavi MN, Khodabandeh G. Prevalence and severity of premenstrual symptoms among Iranian female university students. J Pak Med Assoc. 2009; 59(4):205-8. [In Persian]

[10] Ahmadi J. Prevalence and severity of the Pre-Menstrual Syndrome (PMS) in the last grade high school students of Kerman city. Q J Obstet Nurs. 1993; 18(1):99-101. [In Persian]

[11] Pearlstein T, Stone AB. Premenstrual syndromes. Psychiatry Clin North Am. 1998; 21(3):577-90. [DOI:10.1016/S0193953X(05)70024-1]

[12] Saadati N. Relationship between attitudes towards menstruation with the prevalence of premenstrual syndrome in nursing and midwifery students [MSc. Thesis]. Tehran: Tehran University Medical Sciences; 1993. [In Persian]

[13] Willson R. Obstetrics and gynecology [Kazemi D]. Tehran: Daneshpajo; 1995. [In Persian]

[14] Steiner M, Macdougall M, Brown E. The Premenstrual Symptoms Screening Tool (PSST) for clinicians. Arch Womens Ment Health. 2003; 6(3):203-9. [DOI:10.1007/s00737-003-0018-4] [PMID]

[15] Amiri Farahani L, Farokhi F, Abbasi AA. Prevalence, severity, and clinical manifestations of premenstrual syndrome among the students residing in the dormitories of Arak University of Medical Sciences, Iran. Qom Univ Med Sci J. 2014; 7(6):34-40. [In Persian]

[16] Siahbazi S, Hariri FZ, Montazeri A, Moghaddam Banaem L, Hajizadeh E. Translation and psychometric properties of the Iranian version of the Premenstrual Symptoms Screening Tool (PSST). Payesh. 2011; 10(4):421-7. [In Persian]
[17] Derman O, Kanbur NO, Tokur TE, Kutluk T. Premenstrual syndrome and associated symptoms in adolescent girls. Eur J Obstet Gynecol Reprod Biol. 2004; 116(2):201-6 [DOI:10.1016/j. ejogrb.2004.04.021] [PMID]

[18] Chau JP, Chang AM, Chang AM. Relationship between premenstrual tension syndrome and anxiety in Chinese adolescents. J Adolesc Health. 1998; 22(3):247-9. [DOI:10.1016/S1054139X(97)00206-1]

[19] Perez-Lopez CP, Perez-Roncero G, Lopez-Baena MT, CuadrosLopez JL. Premenstrual syndrome and premenstrual dysphoric disorder: Symptoms and cluster influences. Psychiatry J. 2009; 3:3949. [DOI:10.2174/1874354400903010039]

[20] Kiani Asiabar A, Heidari M, Mohammadi Tabar S, Faghihzadeh S. Prevalence, signs, symptoms and predisposing factors of premenstrual syndromes in employed women. Daneshvar Med. 2009; 16(81):45-54. [In Persian]

[21] Adiguzel H, Taskin EO, Danaci AE. The symptomatology and prevalence of symptoms of premenstrual syndrome in Manisa, Turkey. Turk Psikiyatri Derg. 2007; 18(3):215-22. [In Turkish]

[22] Tabassum S, Afridi B, Aman Z, Tabassum W, Durrani R. Premenstrual syndrome: Frequency and severity in young college girls. J Pak Med Assoc. 2005; 55(12):546-9. [PMID]

[23] Ahmadi F, Naghizadeh M, Direkvandmoghadam A, Mohamadian F, Ghazanfari Z. A study on premenstrual syndromes of high school girl-students in Ilam City (western Iran), 2015. J llam Univ Med Sci. 2018; 26(1):154-63. [In Persian] [DOl:10.29252/sjimu.26.1.154]

[24] Najafi-Sharjabad F, Borazjani F, Avazzadeh Z. Investigation of the prevalence and severity of the premenstrual syndrome and its relationship with exercise among female students in Bushehr University of Medical Sciences. Pajouhan Sci J. 2017; 15(3):43-50. [In Persian] [DOI:10.21859/psj-15037]

[25] Bakhshandehnosrat S, Salehi M, Mobasheri A, Asghari Z, Mohammad Khani $M$. the frequency of clinical symptoms of premenstrual syndrome and premenstrual dysphoric syndrome medical student's (2010). Gorgan Univ Med Sci. 2014, 16(3):127-32. [In Persian]

[26] Alavi A, Salahimoghadam AR, Alimalayeri N, Ramezanpour A. Prevalence of clinical manifestations of premenstrual syndrome and premenstrual dysphoric disorder in students of Bandar Abbas Medical University. Hormozgan Med J. 2007; 10(4):335-41. [In Persian]

[27] Sitwat Z, Abid A, Arif A. Premenstrual Syndrome Symptoms (PMS) and prevalence among university students in Karachi, Pakistan. Int Pharm. 2013; 4(4):113-6. [DOI:10.7897/2230-8407.04420]

[28] Alami McHichi K, Tahiri SM, Moussaoui D, Kadri N. Assessment of premenstrual dysphoric disorder symptoms: Population of women in Casablanca. L'Encephale. 2002; 28(6 Pt 1):525-30. [In French] [PMID] 
This Page Intentionally Left Blank 